\title{
Analysis of the behavior of the supply of drugs for hypertension and diabetes in the state of Rio Grande do Sul between 2006 and 2017 using the ARIMA methodology
}

Antonio do Nascimento Branco ${ }^{1}$ - Universidade Federal de Santa Maria, Depto. Engenharia de Produção

Cristiano Ziegler ${ }^{2}$ - Universidade Federal de Santa Maria, Depto. Engenharia de Produção

Mayara Rohenkohl Ricci ${ }^{3}$ - Universidade Federal de Santa Maria, Depto. Engenharia de Produção

Roselaine Ruviaro Zanini ${ }^{4}$ - Universidade Federal de Santa Maria, Depto. Estatística

Adriano Mendonca Souza ${ }^{5}$ - Universidade Federal de Santa Maria. Dento. Estatística

\section{RESUMO}

O Programa Farmácia Popular é indispensável para o tratamento da hipertensão arterial e do diabetes, as duas principais doenças crônicas não transmissíveis do país. Por esse motivo, esta pesquisa objetivou avaliar o comportamento da oferta desses medicamentos no estado do Rio Grande do Sul. Para isso, métodos estatísticos foram utilizados no banco de dados coletado, sendo a metodologia $\operatorname{ARIMA~}(\mathrm{p}, \mathrm{d}, \mathrm{q})$ a prioritária. Utilizando os métodos de AIC e BIC, foi possível determinar modelo SARIMA $(2,0,0)(1,0,0) 12$ como o melhor para representar o comportamento da oferta desses medicamentos. Os principais resultados apresentam que os últimos três meses influenciam diretamente na quantidade e oferta de medicamentos, com evidências de sazonalidade anual. Além disso, os resultados foram discutidos com outros estudos realizados nesse contexto.

Palavras-chave: Farmácia Popular do Brasil. ARIMA. Medicamentos. Hipertensão. Diabetes.
Editor Responsável: Prof. Dr. Hermes Moretti Ribeiro da Silva

\section{ABSTRACT}

The Popular Pharmacy Program is indispensable for the treatment of hypertension and diabetes, the two principal chronic non-communicable diseases in the country. Therefore, this research aimed to evaluate the behavior of the supply of these drugs in the state of Rio Grande do Sul. For this, statistical methods were used in the collected database, and the ARIMA $(p, d, q)$ methodology was a priority. Using the AIC and BIC methods, it was possible to determine that the SARIMA model $(2,0,0)(1,0,0) 12$ was the best to represent the behavior of the supply of these drugs. The main results presented in the last three months directly influence the quantity and supply of medicines, albeit with annual seasonality. In addition, the results were discussed together with other studies conducted in this context.

Keywords: Popular Pharmacy of Brazil. ARIMA. Medicines. Hypertension. Diabetes.

1.Avenida Roraima, $\mathrm{n}^{\circ}$ 1000, Cidade Universitária, Bairro Camobi, Santa Maria - RS, CEP: 97105-900, antoniobrancov33@gmail.com; 2. cz001097@fahor.com.br; 3. maya.ricci@hotmail.com; 4. rrzanini@smail.ufsm.br; 5. amsouza.sm@gmail.com.

BRANCO, A.N.; ZIEGLER, C.; RICCI, M.R.; ZANINI, R.R.; SOUZA, A.M. Análise do comportamento da oferta de medicamentos contra hipertensão e diabetes no estado do Rio Grande do Sul entre 2006 e 2017 por meio da metodologia ARIMA. GEPROS. Gestão da Produção, Operações e Sistemas, v. 15, n. 2, p. 91 - 110, 2020.

DOI: $10.15675 /$ gepros.v15i2.2435 
Análise do comportamento da oferta de medicamentos contra hipertensão e diabetes no estado do Rio Grande do Sul entre 2006 e 2017 por meio da metodologia ARIMA

\section{INTRODUÇÃO}

O Princípio da universalidade da saúde é uma garantia constitucional expressa no artigo 196 da Constituição Federal. Neste texto, o estado fica obrigado a garantir o acesso indiscriminado à assistência médica, assim como agir por meio de políticas sociais e econômicas no intuito de reduzir o risco de doenças e seus agravos. De maneira concomitante, cabe a ele garantir o acesso universal e igualitário às ações e serviços voltados para a promoção, proteção ou recuperação da saúde (BRASIL, 1988).

A Lei $n^{\circ}$ 10.858/04 criou o Programa Farmácia Popular do Brasil (PFPB) com a finalidade de ampliar o acesso aos medicamentos à população, especialmente à parcela de baixo poder aquisitivo, por meio da comercialização em unidades próprias ou por parcerias com a iniciativa privada (BRASIL, 2004a). Tal programa visa facilitar o acesso ao tratamento das doenças mais recorrentes no país, contribuindo assim para a promoção nacional da saúde.

Segundo dados do Plano Nacional de Saúde (PNS) de 2013, a hipertensão e a diabetes figuram como as principais doenças crônicas não transmissíveis no país. De acordo com o estudo, a hipertensão arterial atinge cerca de 31,3 milhões de pessoas, representando $21,4 \%$ da população. Já a diabetes, atinge em média 9 milhões de pessoas, correspondendo a 6,2\% da população adulta do país (IBGE, 2014).

Outro fator fundamental é a importância da Farmácia Popular no tratamento dessas enfermidades. Segundo o PNS de 2013, no Brasil 35,9\% dos pacientes relataram obter medicamentos para a hipertensão arterial por meio do programa. Em relação aos pacientes com diabetes, esse número apresentou crescimento expressivo, porque 4,2 milhões de pacientes $(57,4 \%$ do total) afirmam também obter medicamentos para a doença nessa farmácia (IBGE, 2014). Desse modo, tais dados ratificam a indispensável necessidade da existência desse programa para a promoção da saúde no país.

No âmbito da mesma pesquisa, o estado do Rio Grande do Sul apresenta o maior percentual de hipertensão da região sul, respondendo por $24,9 \%$ do total. Em relação à diabetes, o estado novamente ganha destaque, correspondendo por $7 \%$ do total nacional. Por sua vez, quando considerado apenas as pessoas que obtiveram no mínimo um medicamento por meio da Farmácia Popular, o estado se destaca para medicamentos anti-hipertensivos com 
Análise do comportamento da oferta de medicamentos contra hipertensão e diabetes no estado do Rio Grande do Sul entre 2006 e 2017 por meio da metodologia ARIMA

45,3\% do total, porém apresenta o menor indicador de obtenção da região sul para os medicamentos contra diabetes (IBGE, 2014).

Por esse motivo, dada a extrema importância deste programa para a promoção da saúde, sobretudo no combate às doenças crônicas já citadas, o presente estudo objetiva avaliar o comportamento da oferta dos medicamentos contra hipertensão e diabetes no Rio Grande do Sul. Para isso, foram utilizados métodos estatísticos univariados baseados no modelo ARIMA a fim avaliar o comportamento dessa oferta, possibilitando assim análises futurais mais acuradas.

A justificativa do desenvolvimento desse estudo, está em aliar um tema de total importância para o Brasil, o Programa Farmácia Popular, e métodos robustos de previsão, ARIMA. O método ARIMA apresenta uma estrutura robusta e acessível, além de ser amplamente utilizada para realizar previsões (CAS, 2018). Com isso, também se pretende colaborar para a construção do conhecimento a respeito do programa e possibilitar, dessa maneira, meios para contribuir com sua manutenção e expansão.

Outro fato que justifica a realização dessa pesquisa, é a possibilidade de identificar os períodos de maior demanda de remédios contra hipertensão e diabetes no estado do Rio Grande do Sul durante o ano, apresentando possíveis sazonalidades. Com esses dados, tomadores de decisão podem adequar o planejamento de estoques para garantir a entrega do produto.

\section{REFERENCIAL TEÓRICO}

\subsection{O programa Farmácia Popular}

O Programa Farmácia Popular foi desenvolvido conjuntamente pelo Ministério da Saúde em parceria com a Fundação Osvaldo Cruz por meio da Lei $\mathrm{n}^{\circ}$ 10.858/04 e do Decreto $\mathrm{n}^{\circ}$ 5.090/04, onde foram instituídas as diretrizes desse programa (BRASIL, 2004b). Segundo as normas citadas, essa iniciativa é fruto de convênio entre os estados, Distrito Federal, municípios, hospitais filantrópicos e a rede privada de drogarias. Sua principal finalidade, segundo Pinto et al. (2011), é atender a parcela da população que não possui recursos para adquirir medicamentos, oferecendo assim produtos básicos e essenciais à saúde a custo subsidiado. 
Análise do comportamento da oferta de medicamentos contra hipertensão e diabetes no estado do Rio Grande do Sul entre 2006 e

2017 por meio da metodologia ARIMA

Desde sua criação, o Programa Farmácia Popular do Brasil sofre alterações no intuído de melhor atender a população. Uma delas é a isenção de medicamentos voltados para doenças crônicas. Desde 2011, a campanha "Saúde Não Tem Preço" isentou do copagamento os medicamentos para o tratamento da hipertensão arterial, diabetes e, posteriormente, asma em ambas as vertentes do programa (SILVA; CAETANO, 2015). No entanto, a modalidade de dispensação com ressarcimento ainda está mantida para o restante dos medicamentos.

Até 2017, o PFPB contava com a comercialização de remédios em unidades próprias, assim como em parceria com o setor privado, vertente denominada “Aqui Tem Farmácia Popular" (ATFP). Atualmente, devido a decisões estratégicas, o programa conta apenas com a vertente conveniada, sendo os recursos destinados para a manutenção das unidades da rede própria repassados integralmente para o ATFP (MINISTÉRIO DA SAÚDE, 2017).

A política do Programa Farmácia Popular especificou uma lista de medicamentos essenciais a serem subsidiados inicialmente pelo governo e fornecidos em farmácias públicas, alguns anos depois, esse programa foi ampliado para farmácias privadas contratadas (EMMERICK et al., 2015). Nas parcerias com as farmácias privadas, o governo cobre $90 \%$ do preço do medicamento, enquanto o paciente paga os $10 \%$ restantes (BERTOLDI et al., 2012).

O Programa Farmácia Popular subsidia 90\% da insulina para diabetes, porém nem todos os medicamentos para diminuir os níveis de glicose (LU et al., 2015). Embora o Programa Farmácia Popular seja uma alternativa para distribuição de medicamentos, o Brasil ainda tem problemas com o acesso. Em 2013, apenas um terço dos pacientes receberam pelo menos um medicamento no serviço público de saúde (MELO et al., 2017).

\subsection{Método de Box e Jenkins para o estudo de séries temporais}

Uma série temporal é uma coleção de observações colhidas sequencialmente ao longo do tempo, apresentando como característica primordial a forte dependência entre os dados vizinhos. Devido a sua especificidade, ela apresenta características peculiares que dificultam sua análise (SOUZA et al., 2010). Dentre elas, de acordo com Moretin e Toloi (1987) estão: presença de tendência, ou seja, a evidência de sentido para a série temporal ao longo dos anos; ocorrência de ciclos e sazonalidade, ou seja, movimentos ondulatórios na série de longa e curta duração respectivamente; assim como a presença de "ruído aleatório" ou erro, abrangendo a variabilidade intrínseca aos dados e que não pode ser modelado. 
Análise do comportamento da oferta de medicamentos contra hipertensão e diabetes no estado do Rio Grande do Sul entre 2006 e 2017 por meio da metodologia ARIMA

Por esse motivo, de acordo com Souza et al. (2010), como a maior parte dos procedimentos estatísticos foi desenvolvida para analisar observações independentes, o estudo baseado em séries temporais necessita da aplicação de metodologias específicas. Nesse contexto, os modelos matemáticos desenvolvidos por de George Box e Gwilyn Jenkins em 1976 figuram como um dos principais métodos aplicados no estudo de séries temporais.

Segundo Souza e Marchezan (2010), o método de Box \& Jenkins consiste na procura por um modelo Autoregressivo Integrado de Médias Móveis (ARIMA), objetivando representar o processo gerador da série temporal. Nesse sentido, os modelos ARIMA são métodos matemáticos que almejam captar o comportamento da correlação presente na série temporal, também denominada "Autocorrelação", a fim de realizar previsões. Os modelos ARIMA são o resultado da combinação de três componentes, também, denominados de "filtros": a componente autoregressiva (AR), o filtro de integração (I) e a componente de médias móveis (MA), sendo essa interação a promotora de vários modelos de previsão (SOUZA et al., 2011).

A utilização do método ARIMA figura como uma poderosa solução para muitos problemas de previsão, segundo Souza (2006), ela proporciona previsões extremamente acuradas para modelos de séries temporais, sendo apropriada para séries de comprimento médio a longo, de preferência com aproximadamente 100 observações. Além de fazer previsões, os modelos de séries temporais são usados para identificar o comportamento das séries ao longo do tempo (SENNA; SOUZA, 2016). Segundo Hikichi, Salgado e Beijo (2017), a construção de modelos Box e Jenkins (1976) é baseada em um ciclo interativo, onde a seleção do modelo apropriado se baseia em sua própria estrutura de dados. Assim, de acordo com esse método, o ciclo é composto por 4 etapas, a saber:

a) Identificação: utilizada para determinar o número de componentes autorregressivos (p), os de médias móveis (q) e da diferença (d);

b) Estimação: etapa em que são estimados os parâmetros do modelo;

c) Verificação: aplicação de testes estatísticos a fim de verificar se os pressupostos da análise são fidedignos. Nesta etapa, caso o modelo investigado não seja apropriado, o ciclo retorna ao início;

d) Previsão: Etapa final do ciclo onde são feitas as inferências futuras utilizando o modelo selecionado como base. 
Análise do comportamento da oferta de medicamentos contra hipertensão e diabetes no estado do Rio Grande do Sul entre 2006 e 2017 por meio da metodologia ARIMA

A utilização desse processo possibilita estimar diversos comportamentos na série, sendo fundamental para a acurácia nas previsões.

\subsubsection{Modelos estacionários e não estacionários}

De acordo com Martins e Werner (2014), a metodologia Box-Jenkins ajuda identificar diferentes modelos ARIMA e entre os melhores estão: o modelo Autorregressivo de ordem $\mathrm{p}$ (AR), o modelo de Médias Móveis de ordem q (MA), o modelo Autorregressivo de Médias Móveis de ordem p e q (ARMA), assim como o próprio modelo Autorregressivo Integrado de Médias Móveis, de ordem p,q com diferença d (ARIMA) que, segundo os autores, figura como o principal caso dentro deste método. Nesse quadro, de acordo com Souza e Marchezan (2010), os modelos AR(p) e MA(p) e ARMA (p,q) apresentam comportamento estacionário.

Por outro lado, para os mesmos autores, o modelo ARIMA (p,d,q) é resultado da aplicação de diferenciação em séries com média inconstante, tornando-as por fim estacionárias. Desse modo, os modelos estacionários e os não estacionários distinguem-se pelo número de diferenças necessárias para estacionarizar uma série, representado pela letra d (SOUZA; MARCHEZAN, 2010).

De acordo com Werner e Ribeiro (2003), no modelo Autorregressivo AR(p) a série de dados históricos $\left(Z_{t}\right)$ é descrita por seus valores passados mais o ruído aleatório $\left(\varepsilon_{t}\right)$, segundo a Equação (1):

$$
\tilde{Z}_{t}=\emptyset_{1} \tilde{Z}_{t-1}+\emptyset_{2} \tilde{Z}_{t-2}+\cdots+\emptyset_{p} \tilde{Z}_{t-p}+\varepsilon_{t}
$$

Onde $\emptyset$ descreve $\left(Z_{t}\right)$ com os valores de $Z_{t-1}$ para $i=1,2, \ldots$, p. Para Souza (2016), vale ressaltar que o modelo só será considerado estacionário se parâmetro $\emptyset$ convergir para $|\emptyset|<1$, também chamada de "condição de estacionalidade". No modelo denominado de Médias Móveis MA(q), por sua vez, a série $\left(Z_{t}\right)$ é resultado da combinação dos ruídos brancos ou aleatórios $\left(\varepsilon_{t}\right)$ do período atual com os anteriores, sendo representado pela Equação (2):

$$
\tilde{Z}_{t}=\varepsilon_{t}+\theta_{1} \varepsilon_{t-1}+\theta_{2} \varepsilon_{t-2}+\cdots+\theta_{q} \varepsilon_{t-q}
$$

Onde $\theta$ descreve como $\left(Z_{t}\right)$ se relaciona com o valor $\varepsilon_{t-i}$ para $\mathrm{i}=1,2, \ldots, \mathrm{q}$ (WERNER; RIBEIRO, 2003). De acordo com Souza (2016), o nome deste modelo vem do fato de que segundo o mesmo autor, o modelo é considerado estacionário se for de ordem finita, assim como o modelo só será considerado estacionário caso $|\theta|<1$, também denominada de "condição de invertibilidade".

GEPROS. Gestão da Produção, Operações e Sistemas, v. 15, nº 2, p. 91 - 110, 2020. 
Análise do comportamento da oferta de medicamentos contra hipertensão e diabetes no estado do Rio Grande do Sul entre 2006 e 2017 por meio da metodologia ARIMA

O Modelo Autorregressivo de Médias Móveis ARMA (p,q), de acordo com Souza e Marchezan (2010), também figuram como modelos estacionários. Segundo eles, este modelo é a união dos métodos supracitados, sendo utilizado em séries encontradas na prática para as quais não se deseja um grande número de parâmetros. Nesse contexto, a notação do modelo $\operatorname{ARMA}(p, q)$ é dada pela Equação 3, a seguir:

$$
\tilde{Z}_{t}=\emptyset_{1} \tilde{Z}_{t-1}+\cdots+\emptyset_{p} \tilde{Z}_{t-p}+\varepsilon_{t}-\theta_{1} \varepsilon_{t-1}-\cdots-\theta_{q} \varepsilon_{t-p}
$$

Onde o parâmetro $\emptyset$ representa a parte autorregressiva; assim como o parâmetro $\theta$, a parte de médias móveis.

Ao mesmo tempo, segundo eles, é perceptível a existência das mesmas condições de estacionalidade e invertibilidade tanto no modelo ARMA (p,d), quanto nas métodos autorregressivos (AR) e de médias móveis (MA), ou seja, $|\emptyset|<1$ e $|\theta|<1$ (NORONHA; ZANINI; SOUZA, 2019).

Em contraste a esses métodos, os modelos lineares não estacionários, baseados em processos lineares homogêneos que não apresentam comportamento explosivo, podem se tornar estacionários por meio de um processo finito de diferenciações $\mathrm{d}$, processo descrito pelos modelos ARIMA (MORETTIN; TOLOI, 1987). Hikichi, Salgado e Beijo (2017) ressaltam que para a correta aplicação do método de Box e Jenkins, há a necessidade da série ser estacionária, requerendo a transformação dos dados originais por meio de diferenças, sendo que, de acordo com eles, 1 ou 2 diferenças são suficientes para estacionalizar séries homogêneas. Nesse sentido, segundo Werner e Ribeiro (2003), o modelo ARIMA (p,d,q) é dado pela Equação (4):

$$
W_{t}=\emptyset_{1} W_{t-1}+\ldots+\emptyset_{p} W_{t-p}+\varepsilon_{t}-\theta_{1} \varepsilon_{t-1}-\ldots-\theta_{q} \varepsilon_{t-q}
$$

Onde novamente parâmetro $\emptyset$ representa a parte autoregressiva; o parâmetro $\theta$ indica a presença de médias móveis, assim como, nesse caso já se subentende a presença da série com diferença.

\subsubsection{Modelos Fracionários}

No contexto da metodologia ARIMA, também é possível modelar séries temporais considerando a possibilidade da diferença (d) poder assumir valores não inteiros, ou seja, fracionários (LIMA; GÓIS; ULISES, 2007). De acordo com Lopes, Macedo e Toyoshima (2016), tal possibilidade é uma maneira natural para conciliar o bom desempenho preditivo de 
Análise do comportamento da oferta de medicamentos contra hipertensão e diabetes no estado do Rio Grande do Sul entre 2006 e 2017 por meio da metodologia ARIMA

curto prazo no modelo ARIMA (p,d,q), com a possibilidade teórica de processos de memória longa, por meio da flexibilização da constante de integração, resultando assim em um modelo Autorregressivo Fracionário de Médias Móveis, ou seja, ARFIMA (p,d,q), descrito pela Equação (5), a seguir:

$$
\emptyset(B) \sum_{k=0}^{\infty}\left(\begin{array}{l}
d \\
k
\end{array}\right)(B)^{k}(c(t)-\bar{c})=\theta(B) \varepsilon_{t}
$$

Onde (B) é o operador de defasagem, $\emptyset$ (B) representa os termos autoregressivos; $\theta$ (B) os de médias móveis; (c) a constante, assim como (ct) representa o ruído branco (HOSKING, 1981). Ao mesmo tempo, segundo o mesmo autor, os valores da diferença (d) fracionária variam entre -1,0 e 1,0 (JIN; FRECHETE, 2004; LIMA; GÓIS; ULISES, 2007).

\subsubsection{Modelos Sazonais}

Considerado as especificidades dos fenômenos abordados em séries temporais, a utilização de outros modelos também se faz necessária. Uma delas, de acordo com Souza (2016), é a ocorrência de periodicidade nos dados indicando a presença de sazonalidade. Segundo o mesmo autor, essa periodicidade pode ser incorporada ao método de Box e Jenkins por meio da utilização de um modelo ARIMA sazonal, assim como é possível realizar diferenças sazonais na série de modo a torná-la estacionária. Para Werner e Ribeiro (2003), ele pode ser incorporado ao modelo SARIMA, porque abrange os métodos que comtemplam séries marcadas por autocorrelação sazonal.

Nesse sentido, segundo Tseng, Yu e Tzeng (2002), a notação do modelo ARIMA sazonal é representada por: $\operatorname{SARIMA}(\mathrm{p}, \mathrm{d}, \mathrm{q})(\mathrm{P}, \mathrm{D}, \mathrm{Q}) \mathrm{s}$, em que $(\mathrm{p}, \mathrm{d}, \mathrm{q})$ representam as ordens autorregressivas e de médias móveis com diferença (ARIMA), assim como (P,D,Q) suas ordens correspondentes do modelo sazonal. Dessa maneira, de acordo com Markridakis et al. (1998) o modelo SARIMA é dado pela Equação (6), a saber:

$$
\left(1-\emptyset_{p} B^{p}\right)\left(1-\phi_{p} B^{s}\right)\left(1-B^{d}\right)\left(1-B^{D}\right) Z_{t}=\left(1-\theta_{q} B^{q}\right)\left(1-\Theta_{Q} B^{s}\right) \varepsilon_{t}
$$

Onde, segundo o autor, a parte anterior da igualdade apresenta os componentes autorregressivos (p), seus componentes sazonais (P), as diferenças (d), assim como as diferenças sazonais (D) consideradas na modelagem proposta. Por outro lado, na outra parte da equação figuram as componentes de médias móveis (q), assim como suas equivalentes da parte sazonal $(\mathrm{Q})$ em períodos determinados (s). 
Análise do comportamento da oferta de medicamentos contra hipertensão e diabetes no estado do Rio Grande do Sul entre 2006 e 2017 por meio da metodologia ARIMA

Contudo, segundo Jacobs (2016), o método SARIMA também apresenta limitações, segundo ele, "um único modelo SARIMA não teria a capacidade de capturar todos os padrões identificados na série". Por esse motivo, o autor recomenda a utilização do ciclo interativo de Box-Jenkins para a identificação do modelo mais adequado.

\subsubsection{Critérios penalizadores para seleção de modelos}

Os critérios penalizadores são utilizados dentro do ciclo interativo de Box-Jenkins, na etapa de identificação de modelos, a fim de encontrar modelos parcimoniosos. Segundo Sobral e Barreto (2011), dada à constatação de que a maioria dos dados reais não se adequam perfeitamente a nenhum modelo matemático, uma das soluções para a resolução deste problema é o aumento de sua ordem. Para os autores, tais problemas de imprecisão podem ser ocasionados por distúrbios nos dados (interferências) ou pela escolha de métodos limitados de modelagem.

Nesse quadro, Akaike (1973) propõe um método para viabilizar o aumento da ordem de um modelo a fim de conseguir uma melhor aderência. Para isso, esta metodologia atribui valores para determinado modelo segundo sua adequação aos dados e a ordem do mesmo, de acordo com a Equação (9), a saber:

$$
A I C_{(p, q)}=\operatorname{Ln} \hat{\sigma}_{p, q}{ }^{2}+\frac{2(p+q)}{n}
$$

Onde $A I C$ é o Critério de Informação de Akaike, $\operatorname{Ln} \widehat{\sigma}_{p, q}{ }^{2}$ é o estimado de máxima verossimilhança do modelo ARMA, $p$ a ordem do modelo Autorregressivo, $q$ a ordem do modelo de Médias Móveis e $n$ o número de observações.

Todavia, não obstante a larga aceitação do Akaike's Information Criterion (AIC) como critério de escolha de modelos, sua utilização pode apresentar falhas em dadas circunstâncias. Por esse motivo, após a contribuição de Akaike (1973), outros métodos complementares também foram elaborados a fim de compensar esta lacuna. Dentre eles, o método mais emitente foi o proposto por Schwarz (1978), sendo utilizando até hoje em conjunto a seu precursor. Nesse contexto, ele é expresso segundo a Equação (10), a saber:

$$
B I C_{(p, q)}=\operatorname{Ln} \hat{\sigma}_{p, q}{ }^{2}+(p+q) \frac{\ln (n)}{n}
$$

Onde BIC é o Critério de Informação Bayesiano, $\hat{\sigma}_{p, q}{ }^{2}$ é o estimador de máxima verossimilhança residual, $\mathrm{p}$ é a ordem do modelo Autorregressivo, q a ordem do modelo de Médias Móveis e n o número de observações. 
Análise do comportamento da oferta de medicamentos contra hipertensão e diabetes no estado do Rio Grande do Sul entre 2006 e 2017 por meio da metodologia ARIMA

\section{PROCEDIMENTOS METODOLÓGICOS}

\subsection{Objeto de estudo}

A presente pesquisa está focada na distribuição de medicamentos pela Farmácia Popular no estado do Rio Grande do Sul, mais especificamente em remédios para o tratamento da hipertensão arterial e diabetes, as duas principais doenças crônicas não transmissíveis do país.

\subsection{Procedimentos de coleta de dados}

A realização deste estudo pode ser caracterizada como pesquisa exploratória de cunho quantitativo. Ao mesmo tempo, para sua constituição, uma ampla revisão bibliográfica foi realizada focando nos temas relacionados à farmácia Popular, assim como as técnicas de previsão envolvendo a metodologia Box-Jenkins. Além disso, algumas bases bibliográficas já consolidadas no meio científico foram consultadas, bem como leis e instruções normativas referentes ao programa na Biblioteca Digital do Senado, bancos de teses de organismos acadêmicos, a Biblioteca Virtual em Saúde (BVS) disponibilizada pelo Ministério da Saúde, bem como o próprio ambiente virtual da FIOCRUZ referente ao PFPB.

Nesse sentido, as principais bases consultadas foram: Scielo, Web of Science e Scopus. Nessa busca, houve preferência aos artigos mais recentes (entre 2005 a 2017), assim como ela foi direcionada pelas interações entre as seguintes palavras-chave: "farmácia popular", "copagamento", "popular pharmacy", “ARIMA", "previsão" e "forecasting”. Paralelamente, a consulta a Biblioteca Virtual em Saúde, a Biblioteca Virtual do Senado e ao ambiente virtual da FIOCRUZ, possibilitou a obtenção de artigos, decretos e manuais diversos, todos referentes ao programa Farmácia Popular.

Posteriormente, valendo-se da Lei de Acesso a Informação Lei $n^{\circ}$ 12.527/2011 (BRASIL, 2011), a obtenção dos dados quantitativos para a realização deste estudo foi feita por meio do Sistema Eletrônico do Serviço de Informações ao Cidadão (e-SIC), abrangendo um horizonte de oferta de medicamentos compreendido entre o final de 2006 ao início de 2017. Foi dada preferência aos medicamentos para o tratamento de hipertensão e diabetes, ofertados pelo programa em todas as cidades do Rio Grande do Sul, compreendendo o referido período citado. 
Análise do comportamento da oferta de medicamentos contra hipertensão e diabetes no estado do Rio Grande do Sul entre 2006 e 2017 por meio da metodologia ARIMA

\subsection{Procedimentos de análise de dados}

De posse de tais dados então, houve o refinamento englobando apenas a quantidade agregada desses medicamentos ofertados por ano. Desse modo, tal resultado propiciou a obtenção de 135 observações, representando o somatório de todas as cidades do estado por ano, possibilitando assim a análise segundo a metodologia ARIMA.

Neste processo, inicialmente o comportamento gráfico da série foi analisada, possibilitando a identificação de sua tendência de crescimento. Posteriormente, seguindo os direcionamentos da referida metodologia, houve a análise da estacionalidade da série por meio do teste de Raízes Unitárias, também conhecido por "Teste Dickey Fuller - ADF".

$\mathrm{Na}$ sequência, foram realizadas inúmeras modelagens objetivando encontrar os modelos mais representativos para descrever o comportamento das séries, ou seja, as ofertas de medicamentos para hipertensão e diabetes no referido estado. Foram identificados os modelos concorrentes para a oferta de medicamentos contra hipertensão e diabetes. Sendo a escolha do modelo mais representativo, realizada considerando os valores de AIC e BIC, segundo os direcionamentos da literatura.

Finalmente, de posse de tais resultados, foi possível entender o comportamento da oferta de medicamentos PFPB para as referidas enfermidades, assim como possibilitou a descoberta de modelos matemáticos que podem viabilizar a realização de previsões neste contexto. Tal fato, então possibilitou realizar discussões a respeito do programa, sua importância social na oferta desses fármacos, sua contribuição ao trabalho já conceituado na literatura especializada, assim como discutir os rumos do Programa Farmácia Popular do Brasil no atual cenário político e econômico nacional.

\section{RESULTADOS E DISCUSSÕES}

A análise dos dados permitiu identificar quais são os medicamentos ofertados para hipertensão e diabetes pelo PFPB, assim como mensurar o aumento dessa oferta ao longo dos anos. Neste contexto, no Quadro 1 são listados os remédios analisados neste estudo, segundo tratamento indicado: 
Análise do comportamento da oferta de medicamentos contra hipertensão e diabetes no estado do Rio Grande do Sul entre 2006 e 2017 por meio da metodologia ARIMA

Quadro 1- Lista de identificação dos medicamentos segundo indicação

\begin{tabular}{|cc|c|}
\hline \multicolumn{1}{|c|}{ HIPERTENSÃO } & DIABETES \\
\hline ABLOK & ENALABAL & CLAMIBEN \\
ANGIPRESS & ENALAMED & CLORIDRATO DE METFORMINA \\
ANGITENS & ENALPRIN & DAONIL \\
ARADOIS & ENAPLEX & GLIBENCLAMIDA \\
ATENOBAL & ENAPROTEC & GLIBENECK \\
ATENOL & GLICAMIN \\
ATENOLAB & HIDROCLOROTIAZIDA & GLICEFOR \\
ATENOLOL & HIDROFLUX & GLICONIL \\
CAPOTRINEO & HIDROLESS & GLIFAGE \\
CAPOX & HIDROMED & GLIFAGE XR \\
CAPTOCORD & GLIONIL \\
CAPTOLAB & LOSARTAN POTÁSSICO & HUMULIN N \\
CAPTOPRIL & HOSARTANA POTASSICA & HUMULIN R \\
CAPTOSEN & MALEATO DE ENALAPRIL & INSUNORM N \\
CLORANA & MALEATO ENALAPRIL & INSUNORM R \\
CLORIDRATO DE PROPANOLOL & MOLOL & MEGUANIN \\
CLORIDRATO DE PROPRANOLOL & PRESSEL & NOVOLIN N \\
CORUS & PRESSOMEDE & NOVOLIN R \\
CYTRANA & PRESSTOPRIL & TEUTOFORMIN \\
DIURIX & RENALAPRIL & TRIFORMIN \\
VALTRIAN & RENITEC & \\
VASOPRIL & RENOPRIL & \\
VASOPRIL & TELOL & \\
ZART & TENOLON & TORLÓS \\
\hline
\end{tabular}

Fonte: Autores (2018).

No entanto, dada as peculiaridades de cada medicamento, sobretudo referente à quantidade especificidades técnicas de dosagem e quantidade, apresenta-se apenas a nomenclatura referência de todos os fármacos disponibilizados pelo programa para o tratamento da hipertensão e diabetes durante o período de análise (2006-2017).

Ao mesmo tempo, após a análise da oferta agregada dos referidos medicamentos no Rio Grande do Sul, foi possível observar a evolução de sua disponibilidade para a população do estado. A análise temporal dos dados demonstra nitidamente a maior oferta de remédios voltados para hipertensão arterial (Figura 1). Fato também justificado pela maior variedade de fármacos direcionados ao tratamento dessa enfermidade, quando comparado aos destinados à diabetes (Quadro 1).

Segundo a Figura 1, desde o início do programa há um nítido crescimento na oferta de medicamentos para o tratamento de ambas as enfermidades, apresentando no ano de $2011 \mathrm{um}$ crescimento acelerado. Em outubro de 2006, foram ofertados 160.465 medicamentos para o combate à diabetes e 561.216 contra a hipertensão arterial. Em fevereiro de 2011, esse valor passou para 1.645.079 de medicamentos hiperglicêmicos e 7.373.351 para os antihipertensivos, ou seja, um crescimento de $925,2 \%$ para medicamentos contra diabetes e 1.213,8\% contra hipertensão. Em abril 2017, a oferta desses fármacos aumentou ainda mais, GEPROS. Gestão da Produção, Operações e Sistemas, v. 15, nº 2, p. 91 - 110, 2020. 
Análise do comportamento da oferta de medicamentos contra hipertensão e diabetes no estado do Rio Grande do Sul entre 2006 e 2017 por meio da metodologia ARIMA

passando para 19.512.472 medicamentos hiperglicêmicos e 57.059.112 anti-hipertensivos, ou seja, um crescimento de $988,4 \%$ e $707,8 \%$ respectivamente.

Figura 1 - Série história de oferta de medicamentos do PFPB no RS

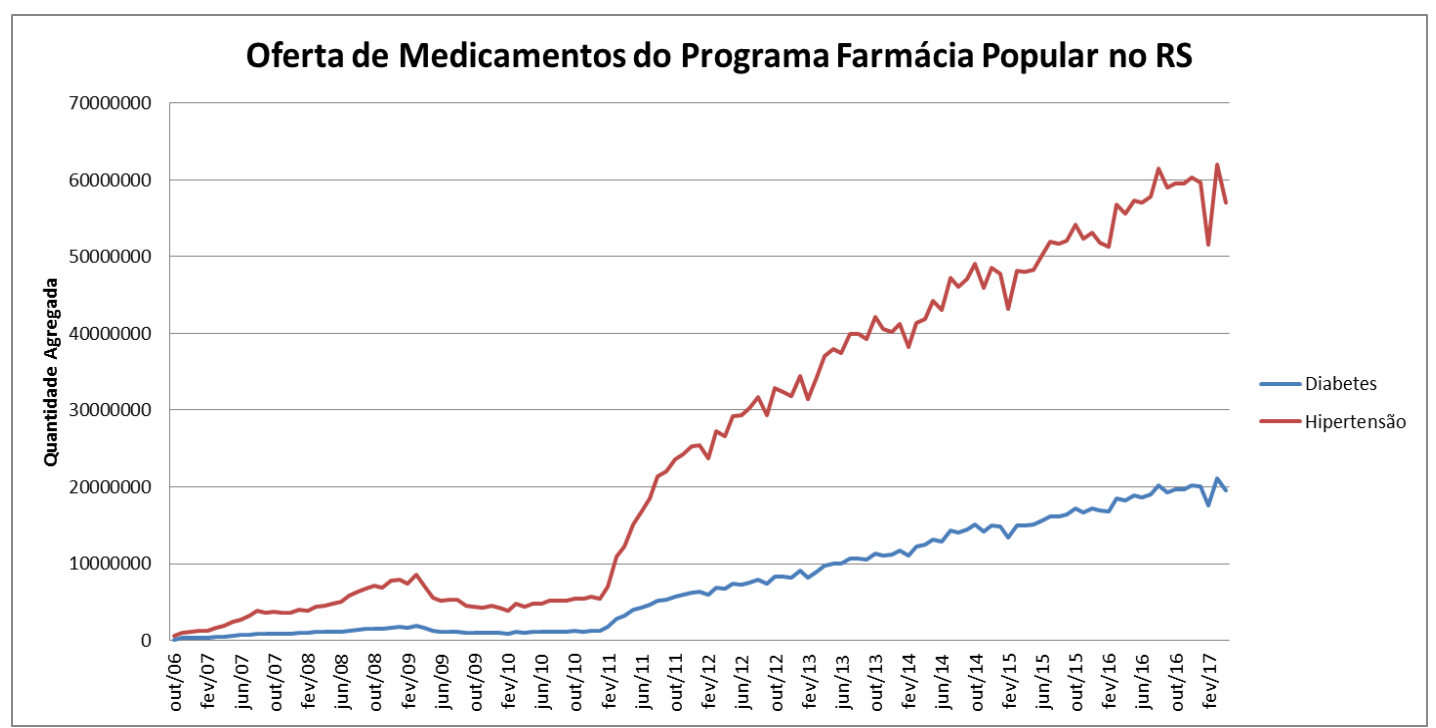

Fonte: Autores (2018).

Em sequência, o comportamento matemático dessa oferta foi investigado iniciando pelo estudo da estacionalidade. Nesse estudo, se constatou a não estacionalidade para ambos os casos, respaldado pela ocorrência de p-valor de 0,4013 para a série de medicamentos para diabetes, assim como de 0,1371 para os fármacos contra hipertensão arterial. Posteriormente, foi possível estacionar ambas as séries diferenças. Em relação a ambos os medicamentos, a aplicação da $2^{\circ}$ diferença propiciou o surgimento do comportamento estacionário das séries ( $\mathrm{p}$ $<0,05)$.

Quando analisada a correlação em nível de cada série de oferta, por meio da Função de Autocorrelação (FAC) e pela Função de Autocorrelação Parcial (FACP), as duas apresentaram comportamento semelhante. Nos dois casos, elas mostram decrescimento lento da FAC e apenas um (o primeiro) lag fora de controle (significativo) na FACP, indicando a presença de um provável comportamento autorregressivo nas duas séries, bem como a ocorrência de períodos fracionários, conforme exposto na Figura 2. 
Análise do comportamento da oferta de medicamentos contra hipertensão e diabetes no estado do Rio Grande do Sul entre 2006 e 2017 por meio da metodologia ARIMA

Figura 2- FAC e FACP em nível das séries de oferta de medicamentos para hipertensão e diabetes

\begin{tabular}{|c|c|c|c|c|c|c|c|c|c|}
\hline \multicolumn{5}{|c|}{ Medicamentos para Diabetes } & \multicolumn{5}{|c|}{ Medicamentos para Hipertensão } \\
\hline & FAC & & FACP & & & FAC & & FACP & \\
\hline 1 & 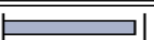 & I & 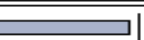 & 1 & I & (" & i & 尸 & 1 \\
\hline 1 & 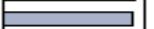 & 10 & 1 & 2 & $\mathbf{I}$ & $\square$ & 1 & 1 & 2 \\
\hline 1 & 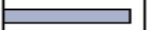 & 10 & 1 & 3 & 1 & 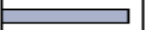 & 10 & 1 & 3 \\
\hline 1 & 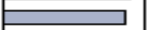 & 10 & I & 4 & I & 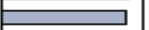 & 10 & 1 & 4 \\
\hline 1 & & 10 & 1 & 5 & 1 & ב & 10 & 1 & 5 \\
\hline 1 & 要 & 1 & 1 & 6 & 1 & 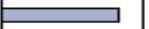 & 1 & 1 & 6 \\
\hline 1 & ए & 1 & I & 7 & 1 & 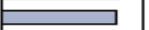 & 1 & 1 & 7 \\
\hline 1 & & & 1 & 8 & 1 & 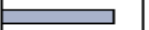 & 1 & I & 8 \\
\hline 1 & ए & 1 & I & 9 & 1 & $\square$ & 1 & 1 & 9 \\
\hline 1 & 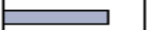 & 10 & I & 10 & I & 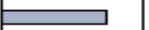 & 10 & 1 & 10 \\
\hline 1 & 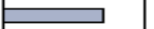 & 10 & 1 & 11 & 1 & $\square$ & 1 & 1 & 11 \\
\hline 1 & 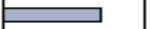 & 1 & 1 & 12 & 1 & 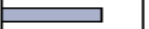 & 1 & 1 & 12 \\
\hline 1 & 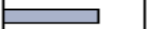 & 10 & I & 13 & 1 & 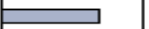 & 1 & 1 & 13 \\
\hline 1 & 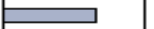 & $1 \mathrm{p}$ & 1 & 14 & 1 & $\square$ & 1 & 1 & 14 \\
\hline 1 & 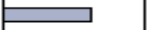 & 10 & 1 & 15 & I & 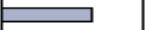 & י & 1 & 15 \\
\hline 1 & 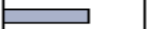 & 1 & 1 & 16 & 1 & 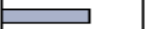 & 1 & 1 & 16 \\
\hline 1 & 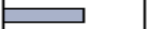 & 1 & 1 & 17 & 1 & $\square$ & 1 & 1 & 17 \\
\hline 1 & 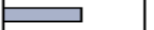 & 10 & 1 & 18 & I & ב & 10 & 1 & 18 \\
\hline 1 & $\longmapsto$ & $1 p$ & 1 & 19 & 1 & 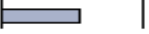 & $1 p$ & 1 & 19 \\
\hline \multicolumn{5}{|c|}{$\begin{array}{l}\text { Date: } 07 / 13 / 17 \text { Time: } 00: 28 \\
\text { Sample: } 1123 \\
\text { Included observations: } 123\end{array}$} & \multicolumn{5}{|c|}{$\begin{array}{l}\text { Date: 08/14/17 Time: 09:14 } \\
\text { Sample: } 1123 \\
\text { Included observations: } 123\end{array}$} \\
\hline
\end{tabular}

Fonte: Adaptado de Eviews 9.0 (2018).

De posse dessas informações então, foi possível estimar, por meio da metodologia de Box-Jenkins para o estudo das séries temporais, os melhores modelos para representar o comportamento estatístico da oferta de medicamentos, tanto para o tratamento da diabetes, como da hipertensão arterial. Como resultado desse processo, a Tabelas 1 foi elaborada agrupando os modelos mais representativos segundo a literatura para cada oferta de medicamentos, ou seja, p-valor inferior a 0,05 e presença de ruídos (erros) não correlacionados (Ruído Branco - R.B.), ambos para hipertensão e diabetes respectivamente.

Conforme é possível observar na Tabela 1, foram identificados 4 (quatro) modelos para os medicamentos anti-hipertensivos e 3 (três) para o hiperglicêmico.

Segundo o direcionamento da literatura, o modelo mais apropriado para representar as duas séries foi o $\operatorname{SARIMA}(2,0,0)(1,0,0) 12$, porque apresentaram os menores valores de AIC e BIC, conforme sugere o referencial teórico supracitado. Desse modo, tal resultado expõe que ambas as séries apresentam dois parâmetros autorregressivos $\left(\emptyset_{1} e \emptyset_{3}\right)$, ou seja, a oferta atual é influenciada pelo horizonte compreendido entre as três últimas quantidades ofertadas. Ao mesmo tempo, foi constatado a influência da sazonalidade na oferta desses produtos, mais especificamente, a influência recorrente os últimos 12 meses na oferta atual. 
Análise do comportamento da oferta de medicamentos contra hipertensão e diabetes no estado do Rio Grande do Sul entre 2006 e

2017 por meio da metodologia ARIMA

Tabela 1 - Modelos concorrentes para oferta de medicamentos contra diabetes

\begin{tabular}{|c|c|c|c|c|c|c|c|}
\hline Modelo & \multicolumn{2}{|c|}{ Coeficientes } & P-Valor (aprox.) & AIC & $\mathrm{BIC}$ & Durbin - Watson & Resíduos Gráficos \\
\hline \multirow{3}{*}{$\operatorname{ARIMA}(1,2,1)$} & $\emptyset 1$ & $-0,45$ & 0,00 & \multirow{3}{*}{28,82} & \multirow{3}{*}{28,89} & \multirow{3}{*}{2,18} & \multirow{3}{*}{ R.B. } \\
\hline & $\theta 1$ & $-0,93$ & 0,00 & & & & \\
\hline & $\emptyset 1$ & $-0,22$ & 0,00 & & & & \\
\hline \multirow[t]{3}{*}{ SARIMA $(2,0,0)(1,0,0) 12$} & $\emptyset 3$ & 0,43 & 0,00 & \multirow[t]{3}{*}{28,41} & \multirow[t]{3}{*}{28,50} & \multirow[t]{3}{*}{1,94} & \multirow[t]{3}{*}{ R.B. } \\
\hline & $\phi 1$ & 0,66 & 0,00 & & & & \\
\hline & $\emptyset 1$ & $-0,23$ & 0,00 & & & & \\
\hline \multirow[t]{3}{*}{ SARIMA $(1,0,1)(1,0,0) 12$} & $\phi 1$ & 0,63 & 0,00 & \multirow[t]{3}{*}{28,42} & \multirow[t]{3}{*}{28,51} & \multirow[t]{2}{*}{1,98} & \multirow[t]{2}{*}{ R.B. } \\
\hline & $\theta 3$ & 0,44 & 0,00 & & & & \\
\hline & D & 0,23 & 0,01 & & & \multirow{3}{*}{2,26} & \multirow{3}{*}{ R.B. } \\
\hline \multirow[t]{2}{*}{$\operatorname{ARFIMA}(2, d, 0)$} & $\emptyset 1$ & 0,48 & 0,00 & \multirow[t]{2}{*}{28,94} & \multirow[t]{2}{*}{29,04} & & \\
\hline & $\emptyset 2$ & 0,52 & 0,00 & & & & \\
\hline \multirow[b]{2}{*}{$\operatorname{ARIMA}(1,2,1)$} & & & 0 & ensăo & \multirow[b]{2}{*}{31,41} & \multirow[b]{2}{*}{2,10} & \multirow[b]{2}{*}{ R.B. } \\
\hline & $\begin{array}{l}\varphi 1 \\
\theta 1\end{array}$ & $\begin{array}{l}-0,41 \\
-0,91\end{array}$ & $\begin{array}{l}0,00 \\
0,00\end{array}$ & 31,34 & & & \\
\hline \multirow{3}{*}{ SARIMA $(1,0,0)(1,0,0) 12$} & $\emptyset 3$ & 0,44 & 0,00 & \multirow{3}{*}{30,89} & \multirow{3}{*}{30,96} & \multirow{3}{*}{2,39} & \multirow{3}{*}{ R.B. } \\
\hline & $\operatorname{SAR}(12)$ & 0,67 & 0,00 & & & & \\
\hline & $\emptyset 1$ & $-0,20$ & 0,00 & & & & \\
\hline \multirow[t]{3}{*}{ SARIMA $(2,0,0)(1,0,0) 12$} & $\emptyset 3$ & 0,45 & 0,00 & \multirow[t]{3}{*}{30,86} & \multirow[t]{3}{*}{30,95} & 1,93 & R.B. \\
\hline & $\phi 1$ & 0,65 & 0,00 & & & & \\
\hline & D & 0,48 & 0,00 & & & & \\
\hline $\operatorname{ARFIMA}(2, \mathrm{~d}, 0)$ & $\varnothing 2$ & 0,47 & 0,00 & 31,33 & 31,42 & 1,58 & R.B. \\
\hline & $\emptyset 3$ & 0,50 & 0,00 & & & & \\
\hline
\end{tabular}

Fonte: Autores (2018).

Portanto, pelas razões apresentadas foi possível descrever os comportamentos matemáticos dessas ofertas, assim como os modelos ideias para a realização de previsões. Assim, segundo o critério de menor AIC e BIC, o modelo escolhido para representar o comportamento das séries de oferta de medicamentos para hipertensão e diabetes foi o $\operatorname{SARIMA}(2,0,0)(1,0,0) 12$.

O ajuste do modelo Box e Jenkins seguiu o um ciclo interativo apresentado por Hikichi, Salgado e Beijo (2017) no Referencial Teórico. O modelo ajustado, SARIMA $(2,0,0)(1,0,0) 12$, apresenta periodicidade, ou seja, sazonalidade nos últimos doze meses, corroborando com os modelos apresentados por Souza (2016). Neste sentido, a oferta de medicamentos deverá ser planejada anualmente, para atender a demanda com eficiência.

A identificação de um modelo com sazonalidade permite adequar os períodos com maior e maior demanda de medicamentos no estado do Rio Grande do Sul. Essa característica confirma a análise de Senna e Souza (2016), que os modelos de séries temporais são usados para identificar o comportamento das séries ao longo do tempo.

A presente pesquisa teve por finalidade ampliar o campo de estudo voltado para a assistência farmacêutica como política pública, mais especificamente o Programa Farmácia GEPROS. Gestão da Produção, Operações e Sistemas, v. 15, nº 2, p. 91 - 110, 2020. 
Análise do comportamento da oferta de medicamentos contra hipertensão e diabetes no estado do Rio Grande do Sul entre 2006 e 2017 por meio da metodologia ARIMA

Popular do Brasil. Nesse sentido, ele pretende expandir as contribuições de Pinto et al. (2011) a respeito da disponibilidade de medicamentos pelo programa, englobando a variável oferta dentro deste contexto. No referido trabalho, os autores abordaram a disponibilidade, o custo e o preço dos medicamentos voltados para hipertensão e diabetes, mais especificamente os fármacos: captopril $25 \mathrm{mg}$ e hidroclorotiazida $25 \mathrm{mg}$, a metformina $500 \mathrm{mg}$ e a glibenclamida 5mg, concluindo que o PFPB apresenta baixa disponibilidade desses medicamentos, apesar de apresentarem o menor custo ao usuário, ratificando assim a grande importância social deste programa.

A baixa disponibilidade de alguns medicamentos, vem ao encontro do exporto por Melo et al. (2017), que apenas 33\% dos pacientes receberam pelo menos um medicamento no serviço público de saúde. Por outro lado, este estudo também vai ao encontro ao produzido por Silva e Caetano (2015), a análise e identificação do modelo matemático representativo da oferta desses medicamentos complementa a análise dos autores da evolução do programa PFPB ao longo dos anos, sobretudo na expansão da disponibilização de medicamentos à população.

\section{CONCLUSÕES}

A análise dos resultados deste estudo atende ao objetivo proposto. A aplicação da metodologia univariada aplicada às séries temporais (ARIMA) possibilitou descobrir o mecanismo gerador das séries de oferta de remédios para hipertensão e diabetes, assim como propiciou a análise mais apurada sobre a maneira como a Farmácia Popular disponibilizava tais medicamentos.

Este estudo trouxe três contribuições importantes para a literatura sobre o Programa Farmácia Popular no Brasil, principalmente acerca de sua relevância no meio social. Como primeira contribuição temos, a grande importância do Programa Farmácia Popular na promoção da saúde, sobretudo no referido estado, dado as quantidades crescentes de oferta para ambos os tratamentos em todos os municípios desta parte do país. Conforme exposto, a hipertensão e a diabetes figuram como as maiores enfermidades não transmissíveis do país, representando um importante desafio para a saúde pública.

A segunda contribuição para a comunidade científica, está nas demonstrações estatísticas, para explicar as influências no comportamento da oferta desses fármacos. 
Análise do comportamento da oferta de medicamentos contra hipertensão e diabetes no estado do Rio Grande do Sul entre 2006 e 2017 por meio da metodologia ARIMA

Conforme constatado, a identificação de um modelo sazonal com apenas dois parâmetros significativos induz à conclusão de que o espaço compreendido entre os últimos três meses influencia diretamente na quantidade a ser ofertada.

A terceira colaboração ao meio acadêmico, está na constatação da ocorrência de sazonalidade anual, influenciando na oferta presente, mais especificamente os últimos doze meses. Assim, tal abordagem pode estar relacionada ao tipo de método de reposição de estoques utilizado pelo programa, assim como às políticas de caráter anual de oferta e precificação dos medicamentos.

No entanto, apesar dos resultados expostos, o referido estudo apresentou relevantes limitações, compreendidas desde a obtenção dos dados até a extrema instabilidade na qual passa o Programa. Ao mesmo tempo, dado o atual cenário político e econômico do país, o Programa Farmácia Popular passa por um período de incertezas podendo, inclusive, ser desarticulado completamente.

Assim, como proposta de abordagem futura, é possível investigar o comportamento da demanda por esses medicamentos, além da maneira como o PFPB realiza suas previsões de demanda e, consequentemente, dimensiona sua oferta. Neste intuito, outra sugestão é analisar a oferta de medicamentos no estado por município, a fim de investigar a maior ou menor prevalência dessas enfermidades por cidade. Ao mesmo tempo, a análise do medicamento mais ofertado por município também é indispensável, uma vez que, após tal descoberta, se devem criar métodos para garantir sua devida disponibilidade nas drogarias do programa.

\section{Referências}

AKAIKE, H. Information Theory and an Extension of the Maximum Likelihood Principle. Springer - Selected Papers of Hirotugu Akaike Nova York, 1973.

BERTOLDI, A. D.; HELFER, A. P.; CAMARGO, A. L; TAVARES, N. U. L.; KANAVOS, P. Is the Brazilian pharmaceutical policy ensuring population access to essential medicines? Globalization and Health, v. 8, n. 1, p.6-15, 2012.

BOX, G. E. P.; JENKINS, G. M. Time series analysis forecasting and control. San Francisco: Holden-Day, 1976.

BRASIL. Constituição (1988). Constituição da República Federativa do Brasil. Brasília, DF: Senado Federal: Centro Gráfico, 1988. 292 p. 
Análise do comportamento da oferta de medicamentos contra hipertensão e diabetes no estado do Rio Grande do Sul entre 2006 e 2017 por meio da metodologia ARIMA

BRASIL. Lei n. 10.858, de 13 de abril de 2004. Autoriza a Fundação Oswaldo Cruz - Fiocruz a disponibilizar medicamentos, mediante ressarcimento, e dá outras providências. Diário Oficial da União, Brasília, DF, 14 abr. 2004a.

BRASIL. Lei $n^{\circ} 5.090$ de 20 de maio de 2004. Institui o programa "Farmácia Popular do Brasil", e dá outras providências. Diário Oficial da União, Brasília, DF, 21 mai. 2004b.

BRASIL. Lei $\mathrm{n}^{\circ} 12.527$ de 18 de novembro de 2011. Regula o acesso a informações previsto no inciso XXXIII do art. 5o, no inciso II do $\S 3^{\circ}$ do art. 37 e no $\S 2^{\circ}$ do art. 216 da Constituição Federal; altera a Lei $\mathrm{n}^{\circ}$ 8.112, de 11 de dezembro de 1990; revoga a Lei $\mathrm{n}^{\circ}$ 11.111, de 5 de maio de 2005, e dispositivos da Lei ${ }^{\circ} 8.159$, de 8 de janeiro de 1991; e dá outras providências. Diário Oficial da União, Brasília, DF, 18 nov. 2011.

CAS, C. G. Application of The ARIMA Model to Forecast the Price of the Commodity Corn. GEPROS. Revista Gestão da Produção Operações e Sistemas, v. 11, n. 1, p.263-279, 2018.

EMMERICK, I. C. M.; NASCIMENTO, J. M.; PEREIRA, M. A.; LUIZA, V. L.; ROSSDEGNAN, D. Farmácia Popular Program: changes in geographic accessibility of medicines during ten years of a medicine subsidy policy in Brazil. Journal of Pharmaceutical Policy and Practice, v. 8, n. 1, p.1-10, 2015.

FAVA, V. L. Manual de econometria. In: VASCONCELOS, M. A. S.; ALVES, D. São Paulo: Editora Atlas, 2000.

HIKICHI, S. E.; SALGADO, E.G.; BEIJO, L. A. Forecasting number of ISO 14001 certifications in the Americas using ARIMA models. Journal of Cleaner Production, v. 147, n. 20, 2017.

HOSKING, J. R. Fractional differencing. Biometrika, v. 68, n. 1, 1981.

INSTITUTO BRASILEIRO DE GEOGRAFIA E ESTATÍSTICA. IBGE. Pesquisa Nacional de Saúde 2013: Percepção do estado de saúde, estilos de vida e doenças crônicas. Rio de Janeiro, 2014.

JACOBS, W. Combination of Box-Jenkins and MLP/RNA models for forecasting. IEEE Latin America Transactions, v. 14, n. 4, 2016.

JIN, H. J.; FRECHETE, D. L. Fractional integration in agricultural futures prices volatilities. American Journal of Agricultural Economics, v. 86, n. 2, 2004.

LIMA, R. C.; GÓIS, M. R.; ULISES, C. Previsão de preços futuros de Commodities Agrícolas com diferenciações inteira e fracionária, e erros heteroscedásticos. Revista de Economia e Sociologia Rural, v. 45, n. 3, 2007. 
Análise do comportamento da oferta de medicamentos contra hipertensão e diabetes no estado do Rio Grande do Sul entre 2006 e 2017 por meio da metodologia ARIMA

LOPES, L. S.; MACEDO, L. R.; TOYOSHIMA, S. H. Integração Fracionária nos Ciclos Econômicos de Longo Prazo no Brasil: Evidências Iniciais de Criticalidade Auto-Organizada. Revista Brasileira de Economia, v. 70, n. 3, 2016.

LU, C. Y., EMMERICK, I. C. M.; STEPHENS, P.; ROSS-DEGNAN, D.; WAGNER, A. K. Uptake of new antidiabetic medications in three emerging markets: a comparison between Brazil, China and Thailand. Journal of Pharmaceutical Policy and Practice, v. 8, n. 1, p.1$8,2015$.

MAKRIDAKIS, S.; WHEELWRIGHT, S. C.; HYNDMAN, R. J. Forecasting: Methods and Applications. 3. ed. New York: John Wiley \& Sons, 1998.

MARTINS, V.L.M., WERNER, L. Comparação de previsões individuais e suas combinações: um estudo com series industriais. Production, v. 24, n. 3, 2014.

MELO, A. C.; GALATO, D.; MANIERO, H. K.; FRADE, J. C. Q. P.; PALHANO, T. J.; DA SILVA, W. B.; JOÃO, W. D. S. J. Pharmacy in Brazil: progress and challenges on the road to expanding clinical practice. The Canadian Journal of Hospital Pharmacy, v. 70, n. 5, p. $381,2017$.

MINISTÉRIO DA SAÚDE. Sobre o programa. 2017. Disponível em:< http://portalms.saude.gov.br/acoes-e-programas/farmacia-popular/sobre-o-programa>. Acesso em $16 / 09 / 2018$.

MORETTIN, P.A.; TOLOI, C.M. C. Previsão de series temporais. São Paulo: Atual, 1987.

NORONHA, M. O.; ZANINI, R. R.; SOUZA, A. M. The impact of electric generation capacity by renewable and non-renewable energy in Brazilian economic growth. Environmental Science and Pollution Research, p.1-24, 2019.

PINTO, C. D. S.; COSTA, N. R.; CASTRO, C. G. O. Quem acessa o Programa Farmácia Popular do Brasil? Aspectos do fornecimento público de medicamentos. Revista Ciência \& Saúde Coletiva, v. 16, n.6, 2011.

SCHWARZ, G. Estimating the dimension of a model. The Annals of Statistics, v. 6, n. 2, 1978.

SENNA, V.; SOUZA, A. M. Assessment of the relationship of government spending on social assistance programs with Brazilian macroeconomic variables. Physica A: Statistical Mechanics and its Applications, v. 462, p.21-30, 2016.

SILVA, R. M.; CAETANO, R. Programa "Farmácia Popular do Brasil": caracterização e evolução entre 2004-2012. Ciência \& Saúde Coletiva, v. 20, n.10, 2015.

SOBRAL, T. E. L.; BARRETO, G. Análise dos critérios de informação para a seleção de ordem em modelos autoregressivos. In: $10^{\circ}$ CONFERÊNCIA BRASILEIRA DE 
Análise do comportamento da oferta de medicamentos contra hipertensão e diabetes no estado do Rio Grande do Sul entre 2006 e 2017 por meio da metodologia ARIMA

DINÂMICA, CONTROLE E APLICAÇÕES. 4., 2011. Anais... DINCON, Águas de Lindóia, SP,2011.

SOUZA, A. M.; SOUZA, F. M.; FERREIRA, N.; MENEZES, R. Eletrical energy supply for Rio Grande do Sul, Brazil, using forecast combination of weighted eigenvalues. GEPROS. Revista Gestão da Produção Operações e Sistemas, n. 3, p. 23-39, 2011.

SOUZA, A.; MARCHEZAN, A. Previsão do preço dos principais grãos produzidos no Rio Grande do Sul. Ciência Rural, v. 40, n. 11, 2010.

SOUZA, F. M.; ALMEIDA, S. G. D.; GUARNIERI, J. P.; SOUZA, A. M.; LOPES, L. F. D. Previsão do consumo de cimento no estado do rio grande do sul. Pesquisa Operacional para o Desenvolvimento, v. 2 n. 1, 2010.

SOUZA, F. M. Modelos Box e Jenkins aplicados à previsão de demanda de leitos hospitalares. 2006. 83 p. Monografia (Especialização) - Universidade federal de Santa Maria, Santa Maria, RS, 2006.

SOUZA, F. M. Modelos De Previsão: aplicações à energia elétrica - ARIMA- ARCH-AI e ACP. Curitiba: Appris, 2016.

TSENG, F. M.; YU, H. C.; TZENG, G. H. Combining neural network model with seasonal time series ARIMA model. Technological Forecasting and Social Change, v. 69, n. 1, p.7187, 2002.

WERNER, L.; RIBEIRO, J. L. D. Previsão de demanda: uma aplicação dos modelos BoxJenkins na área de assistência técnica de computadores pessoais. Gestão \& Produção, v.10, n.1, 2003 . 